\title{
Editorial: Special Issue on Vascular Access
}

\author{
Towards Improving Vascular Access
}

Chronic kidney disease (CKD) refers to an irreversible, progressive reduction of kidney function. This condition is generally asymptomatic except in its later stages where it is often referred to as end stage renal disease (ESRD). High blood pressure, diabetes, or poisoning can lead to a loss of renal function reducing the ability of the kidneys to filter blood. When there is a significant loss of renal function the ESRD patient will be referred to a replacement therapy such as dialysis or transplant. The cumulative global cost for treatment (dialysis and transplantation) of ESRD patients is predicted to exceed $€ 750$ Billion in the next decade. ${ }^{17}$ Dialysis treatments alone account for $2 \%$ of national healthcare budgets in developed countries and this is expected to double in the next 5 years. ${ }^{16}$

For those patients with ESRD the options for renal replacement therapy are transplantation, peritoneal dialysis, or hemodialysis. Transplantation is restricted by the lack of donor organs and peritoneal dialysis is suitable for only a minority of patients. Hemodialysis (HD) therefore remains the most common treatment modality and requires creation of an access site in the arm of patients, a process that suffers from six month failure rates of up to $50 \%{ }^{7}$ Treatment of failing access sites is reviewed by Inston et al. ${ }^{8}$ in this special issue. They report the clinical perspective on methods available to treat a failing access. Their paper highlights how even though radiological intervention has become the first choice therapy, the future of vascular access is in better understanding of the disease formation process so that early detection of potential vascular access failure is possible, leading to early intervention and increased vascular access patency. Importantly, as the number of patients on dialysis today is almost double that of 15 years ago, and is projected to double again in the next decade, improvements in the creation of access sites in HD patients are urgently required.

To that end the desired outcome for adequate and efficient hemodialysis is a reliable vascular access which is easily accessible and provides consistently high flow rates greater than $600 \mathrm{ml} / \mathrm{min} .^{12,14}$ The three methods of achieving high flow dialysis venous access are arteriovenous fistula (AVF) formation, arteriovenous graft (AVG) implantation, or central venous catheter (CVC) insertion. AVF and AVG methods are limited by the formation of vessel narrowing (stenosis) due to venous intimal hyperplasia (VIH) or the access fails to mature.
These result in poor dialysis, recirculation, bleeding, and ultimately vessel occlusion due to thrombosis.

In this special issue Lee, ${ }^{9}$ presents a nephrologist's perspective on the Fistula First Initiative in the US and how this initiative has affected patient treatment. This paper reviews the treatment choices available to clinicians and how that choice is made. The paper highlights how recent moves have been towards a Fistula First Catheter Last approach and the near future will move towards a Right Access for Right Patient Approach. It is expected that with increased understanding of access failure mechanisms, improved access patency will be possible and will positively affect patient treatment and quality of life. AVF is currently the preferred access type due to its superior patency rates and fewer complications compared with arteriovenous grafts and catheters ${ }^{1}$ and therefore, this special issue focuses on AVF. Fistula non-maturation and venous stenosis are the primary contributors to access failure. An AVF is deemed mature if, after 6 weeks, it supports a flow of $600 \mathrm{~mL} / \mathrm{min}$, it is located $6 \mathrm{~mm}$ from the surface of the skin and has a diameter greater than $6 \mathrm{~mm} .{ }^{13}$ To assess AVF function various imaging modalities are available to clinicians. In this special issue Murphy et al. ${ }^{10}$ highlight the clinically available imaging modalities employed to assess vascular access sites both pre and post access creation. Advantages and disadvantages of each are discussed and the future direction of each imaging modality is presented. Also evident from this paper is how such imaging modalities could be employed in the next steps in vascular access research, from highlighting the optimum access location on a patient specific basis to surveillance of the access post-operatively.

Maturation and arterialisation on the venous side of the junction are required for successful completion of the procedure, as flow rates are required to be high. While enlargement and outward remodelling is required for initiation of haemodialysis, to sustain the junction, inward vessel narrowing must be limited. Inadequate dilation or outward remodelling is a leading cause of maturation failure and is a factor that is often overlooked. Venous stenosis is the other leading cause of failure, a form of inward remodelling which is characterised by abnormal intimal hyperplasia which reduces the lumen area. ${ }^{15}$ The abnormal hemodynamics arising from fistula creation are believed to 
provide a stimulus for both facets of remodelling. In this special issue Remuzzi et al. ${ }^{11}$ present how the maturation failure cascade (and the key biological cues that result in either a success or failure to mature of the vascular access) remains poorly elucidated. Also demonstrated is how arterial-type hemodynamics affect venous wall cells requires significant additional research. Further, once these cells have experienced the particular mechanical cue that initiates the disease formation cascade, questions arise as to what pathways are activated that lead an access to fail.

It is generally agreed that many of the vessel responses are directly linked to mechanosensors at the vessel wall. Several metrics have been derived to represent the shear stress that blood imposes on the endothelial cells that line the inside of the vessel wall, with high, low, oscillatory, temporal, gradient, and time averaged wall shear stress all utilised to try to link flow and access failure. In this special issue Cunnane et $\mathrm{al}^{2}{ }^{2}$ present a review of differing hemodynamic parameters available through the use of in silico models and how some of these metrics have been used previously. The diversity of the hypotheses that exists in the literature to date evidences the fact that the exact physiological response that leads to the initiation of the disease formation cascade remains poorly understood.

Also, in this special issue Ene-Iordache et al. ${ }^{4}$ investigate how idealised in silico modelling of vascular access contributes to the understanding of vascular access failure. Idealised in silico models are powerful tools that can be employed to investigate the effects of changing geometrical or hemodynamic parameters in a fast an efficient manner. Recent trends of moving towards patient specific models, while important and necessary, do not exclude the usefulness of idealised models in the future challenges of improving vascular access. The paper also highlights how, as a community, standardisation of modelling approaches will help advance our knowledge of vascular access failure. Regarding the use of realistic geometries, in this special issue Grechya et al. ${ }^{6}$ present an example of how in silico models can be employed to investigate surgical approaches to the formation of AVFs employing realistic geometries and virtual surgery. In particular, this study demonstrates how in silico models could be employed in the future to optimise the AVF junction geometry once the key hemodynamic parameter that stimulates the access failure cascade has been identified. In this paper the authors investigate both wall shear stress and oxygen flux in the junction area and demonstrate how these vary depending on the AVF construction approach. The paper concludes with suggestions on how future simulations can be further improved to increase modelling accuracy to the in vivo situation.
In silico models require validation of their results and in this special issue Drost et al. ${ }^{3}$ review the importance of experimental studies as standalone studies in their own right and also as validation of in silico models. Methods available to achieve such validation are presented and limitations highlighted. In particular, the role of in silico models to generate hemodynamic parameter distributions is a significant advancement in our understanding of what might be triggering endothelial cells to initiate the failure to mature process or the intimal hyperplasia process. Such parameter distributions cannot be obtained from experimental studies. Importantly, velocity profiles within the access area can be measured experimentally and subsequently used to validate an in silico model which can then be further interrogated for differing hemodynamic parameter distributions. Recent advances in experimental measurements of flow are also discussed and how these can be leveraged in future research in this area to positively affect the progress in this research field.

Finally, in this special issue Franzoni et al. ${ }^{5}$ review in vitro studies that have attempted to determine the key hemodynamic parameter that negatively affects the maturation process or leads to intimal hyperplasia. To date such parameters have not been identified conclusively and suggestions as to how this research approach can be progressed are put forward.

What this special issue highlights in particular is the complexity of the problems created when the venous system is exposed to arterial type flow. To achieve the desired goal of increased patency rates for vascular access, nephrologists, renal transplant surgeons, vascular surgeons, biomedical engineers and cellular biologists all have to work more closely together and enact a truly multi-disciplinary approach to solving this multi-faceted research problem. This special issue provides review papers on the key aspects of research on vascular access from all of these perspectives. The nature of vascular access and the multidisciplinary approach required to improve the patency of vascular access is evident from the contributions.

Understanding the mechanisms which lead to successful maturation or failure will help improve current surveillance strategies and will lead to new targets and therapies for intervention to maximise access patency. Increasing our understanding of mechanisms that stimulate VIH will allow further research on surgical interventions which will impact on vascular access patency rates and in turn patients will benefit both in quality of life and survival.

We would like to thank the editor for the opportunity to put together this special issue and would particularly like to thank the contributors for their collaborative efforts in putting this special issue together. 


\section{ACKNOWLEDGMENTS}

Michael T. Walsh's efforts in putting this special issue together was supported by the European Union's Seventh Framework Programme for research, technological development, and demonstration under Grant Agreement No. 324487 (ReDVA). James E. Moore Jr. would like to acknowledge support from the Royal Academy of Engineering, The Sir Leon Bagrit Trust, and a Royal Society Wolfson Research Merit Award.

\section{REFERENCES}

${ }^{1}$ Besarab, A. Resolved: Fistulas are preferred to grafts as initial vascular access for dialysis. Pro. J. Am. Soc. Nephrol. (JASN) 19(9):1629-1631, 2008.

${ }^{2}$ Cunnane, C. V., E. M. Cunnane, and M. T. Walsh. Cardiovasc. Eng. Technol., 2017. doi:10.1007/s13239-017-0307-0.

${ }^{3}$ Drost, S., N. Alam, J. G. Houston, and D. N. Newport. Cardiovasc. Eng. Technol., 2017. doi:10.1007/s13239-0170311-4.

${ }^{4}$ Ene-Iordache, B., and A. Remuzzi. Blood flow in idealized vascular access for hemodialysis: a review of computational studies. Cardiovasc. Eng. Technol., 2017. doi: 10.1007/s13239-017-0318-x.

${ }^{5}$ Franzoni, M., and M. T. Walsh. Towards the identification of hemodynamic parameters involved in arteriovenous fistula maturation and failure: a review. Cardiovasc. Eng. Technol., 2017. doi:10.1007/s13239-017-0322-1.

${ }^{6}$ Grechya, L., F. Iori, R. W. Corbett, W. Gedroycc, N. Duncan, C. G. Caro, and P. E. Vincent. The effect of arterial curvature on blood flow in arterio-venous fistulae: realistic geometries and pulsatile flow. Cardiovasc. Eng. Technol., 2017. doi:10.1007/s13239-017-0321-2.

${ }^{7}$ Huijbregts, H. J. T., M. L. Bots, C. H. A. Wittens, Y. C. Schrama, F. L. Moll, P. J. Blankestijn, and on behalf of the CIMINO Study Group. Hemodialysis arteriovenous fistula patency revisited: results of a prospective, multicenter initiative. Clin. J. Am. Soc. Nephrol. 3(3):714-719, 2008.

${ }^{8}$ Inston, N., J. Al Shakarchi, A. Khawaja, and R. Jones. Maintaining patency of vascular access for haemodialysis. Cardiovasc. Eng. Technol., 2017. doi:10.1007/s13239017-0320-3.

${ }^{9}$ Lee, T. Fistula first initiative: historical impact on vascular access practice patterns and influence on future vascular access care. Cardiovasc. Eng. Technol., 2017. doi: 10.1007/s13239-017-0319-9.
${ }^{10}$ Murphy, E. A., R. A. Ross, R. G. Jones, S. J. Gandy, N. Aristokleous, M. Salsano, J. R. Weir-McCall, S. Matthew, and J. G. Houston. Imaging in vascular access. Cardiovasc. Eng. Technol., 2017. doi:10.1007/s13239-0170317-y.

${ }^{11}$ Remuzzi, A., and M. Bozzetto. Biological and physical factors involved in the maturation of arteriovenous fistula for hemodialysis. Cardiovasc. Eng. Technol., 2017. doi: 10.1007/s13239-017-0323-0.

${ }^{12}$ Shenoy, S. Innovative surgical approaches to maximize arteriovenous fistula creation. Semin. Vasc. Surg. 20(3):141-147, 2007.

${ }^{13}$ Sidawy, A. N., L. M. Spergel, A. Besarab, M. Allon, W. C. Jennings, F. T. Padberg, M. H. Murad, V. M. Montori, A. M. O'Hare, K. D. Calligaro, R. A. Macsata, A. B. Lumsden, and E. Ascher. The Society for Vascular Surgery: clinical practice guidelines for the surgical placement and maintenance of arteriovenous hemodialysis access. J. Vasc. Surg. 48(5 Suppl):2S-25S, 2008.

${ }^{14}$ Tordoir, J., B. Canaud, P. Haage, K. Konner, A. Basci, D. Fouque, J. Kooman, A. Martin-Malo, L. Pedrini, F. Pizzarelli, J. Tattersall, M. Vennegoor, C. Wanner, P. ter Wee, and R. Vanholder. EBPG on vascular access. Nephrol. Dial. Transplant. 22(Suppl 2):ii88-ii117, 2007.

${ }^{15}$ Wang, Y., M. Krishnamoorthy, R. Banerjee, J. Zhang, S. Rudich, C. Holland, L. Arend, and P. Roy-Chaudhury. Venous stenosis in a pig arteriovenous fistula model-anatomy, mechanisms and cellular phenotypes. $\mathrm{Ne}$ phrol. Dial. Transplant. 23(2):525-533, 2008.

${ }^{16}$ www.ekha.eu.

${ }^{17}$ www.worldkidneyday.org.

Michael T. WALSH

Health Research Institute, Bernal Institute,

School of Engineering,

University of Limerick, Limerick,

Ireland

Electronic mail: Michael.Walsh@ul.ie

James E. Moore JR.

Department of Bioengineering

Imperial College London,

London SW7 2AZ, UK

Electronic mail: james.moore.jr@imperial.ac.uk 